Portland State University

PDXScholar

\title{
Recommendations for Integrating Peer Mentors in Hospital-Based Addiction Care
}

\author{
Honora Englander \\ Oregon Health \& Science University \\ Jessica Gregg \\ Oregon Health \& Science University \\ Janie Gullickson \\ Mental Health \& Addiction Association of Oregon \\ Onesha Cochran-Dumas \\ Mental Health \& Addiction Association of Oregon \\ Chris Colasurdo \\ Pacific Northwest University of Health Sciences
}

See next page for additional authors

Follow this and additional works at: https://pdxscholar.library.pdx.edu/socwork_fac

Part of the Social Work Commons

Let us know how access to this document benefits you.

\begin{abstract}
Citation Details
Published as: Honora Englander, Jessica Gregg, Janie Gullickson, Onesha Cochran-Dumas, Chris Colasurdo, Juliet Alla, Devin Collins \& Christina Nicolaidis (2019). Recommendations for integrating peer mentors in hospital-based addiction care. Substance Abuse, 1-6.
\end{abstract}

This Post-Print is brought to you for free and open access. It has been accepted for inclusion in Social Work Faculty Publications and Presentations by an authorized administrator of PDXScholar. Please contact us if we can make this document more accessible: pdxscholar@pdx.edu. 


\section{Authors}

Honora Englander, Jessica Gregg, Janie Gullickson, Onesha Cochran-Dumas, Chris Colasurdo, Juliet Alla, Devin Collins, and Christina Nicolaidis 
Title: Recommendations for integrating peer mentors in hospital-based addiction care Authors: Honora Englander $\mathrm{MD}^{1}$ Jessica Gregg MD PhD ${ }^{1}$ Janie Gullickson, MPA ${ }^{2}$ Onesha Cochran Dumas ${ }^{2}$ Chris Colasurdo ${ }^{2,3}$ Devin Collins, $\mathrm{MA}^{1,4}$ Juliet Alla ${ }^{1}$ Christina Nicolaidis MD MPH ${ }^{1,5}$

\section{Affiliations:}

1. Oregon Health \& Science University, Portland, OR

2. Mental Health America of Oregon, Portland, OR

3. Pacific Northwest University of Health Sciences, Yakima, WA

4. University of Washington Department of Sociology, Seattle, WA

5. Portland State University School of Social Work, Portland, OR

\section{Corresponding author:}

Honora Englander, MD, FACP

BTE 119, Oregon Health \& Science University

3181 SW Sam Jackson Park Road

Portland, Oregon 97239

Phone 503-494-8311

englandh@ohsu.edu

Acknowledgements: Authors would like to thank Alisa Patten for her support in preparing this manuscript, and the entire IMPACT clinical and research team for their support of peers. Dr Englander would like to thank Dr David Bangsberg for his mentorship in developing this manuscript.

Funding: This work was funded by Oregon Health \& Science University. OHSU had no role in the design and conduct of the study; collection, management, analysis, and interpretation of the data; preparation, review, or approval of the manuscript; and decision to submit the manuscript for publication.

\section{Author Contributions: All authors made significant contributions to this work. $\mathrm{Dr}$} Englander, Dr Gregg, Ms Gollickson, Ms Cochran, Mr Colasurdo, and Dr Nicolaidis were involved in conception and design, summarizing lessons learned, developing recommendations, writing and revision of the manuscript. Ms Alla and Mr Collins were involved in conception and design, writing and revision of the manuscript.

Conflict of Interest: No authors have any conflicts of interest to disclose

Word Count: Manuscript 3181; Abstract 262

Exhibits: 1 table 


\section{Abstract}

Legislators and health systems have recently begun to explore the use of peer mentors as part of hospital-based addiction teams. Integrating peers into hospitals is a complex undertaking still in its infancy. Peers' lived experience of addiction and its consequences, combined with their distance from medical culture and hierarchy, is at the core of their power - and creates inherent challenges in integrating peers into hospital settings. Successful integration of peers in hospitals has unique challenges for individual providers, health systems, and the peers themselves. We have included peers as part of a hospital-based addiction medicine team at our hospital since 2015. In this manuscript, we outline some unique challenges, share lessons learned, and provide recommendations for integrating peers into hospital-based SUD care.

Challenges include the rigid professional hierarchy of hospitals which contrasts with peers' role, which is built on shared life experience and relationship; different expectations regarding professional boundaries and sharing personal information; the intensity of the hospital environment; and, illness severity of hospitalized people which can be emotionally draining and increase peers' own risk for relapse.

Recommendations focus on establishing a way to finance the peer program, clearly defining the peer role, creating a home base within hospital settings, creating a collaborative and structured process for hiring and retaining peers, identifying peers who are likely to succeed, providing initial and ongoing training to peers that extends beyond typical peer certification, ways to introduce peer program to hospital staff, and providing regular, meaningful supervision.

We hope that our recommendations help other hospital systems capitalize on the practical lessons learned from our experience. 


\section{Background}

Peer mentorship and mutual-aid based support have a long history in addiction care and recovery services. Recognizing the power of peer mentorship, many care settings, including substance use disorder treatment centers, primary care, and emergency departments, now integrate peers into substance use disorder (SUD) treatment teams. ${ }^{1,2}$ Researchers have shown that inclusion of peer mentors in SUD treatment improves outcomes, including reduced relapse rates; increased treatment retention; improved relationships with treatment providers; and increased satisfaction with treatment experience., ${ }^{3,4}$

Building on these successful outcomes, legislators and health systems have recently begun to explore the use of peers in hospital-based addiction treatment as well. ${ }^{5,6}$ However, integrating peers into hospital care is a complex undertaking still in its infancy. Peers' lived experience of addiction and its consequences, combined with their distance from medical culture and hierarchy, is at the core of their power - and creates inherent challenges in integrating peers into hospital settings.

The peer role is fundamentally different from conventional medical providers. Unlike conventional therapeutic relationships, peer mentorship relies on the principles of mutuality and reciprocity of two equals who share similar experiences. ${ }^{7}$ Principles of peer support emphasize the importance of connection, equally shared power, and an understanding and acceptance of different worldviews and choices. ${ }^{8}$

Our experience including peers in a hospital-based addiction medicine team shows that peers can play a potentially critical role in building trust ${ }^{9,10}$ and in developing trauma-informed, patient-centered systems that can respond to the needs of hospitalized adults with SUD. ${ }^{10}$ Peer mentors can be particularly valuable for hospitalized patients with SUD, who often feel judged, discriminated against, and unwelcome in hospital settings. Conflict and communication gaps between patients with SUD and hospital providers are common, and can lead to mistrust and tensions that may result in poor patient outcomes (including patients leaving against medical advice) and provider distress. ${ }^{11}$ Peers - who walk in both worlds of person with SUD and part of the healthcare team - can bridge this divide.

We are champions for the spread of hospital-based peer interventions. However, successful integration of peers into hospital care has unique challenges for individual providers, health systems, and the peers themselves. While literature describes factors to support peer integration into primary care and SUD treatment settings, little is known about how peer mentors may be best integrated into hospital care. Anticipating and addressing the challenges of peers in hospital settings will be important to their success and to sustaining commitment to spread of hospital-based peer interventions.

This commentary aims to outline some unique challenges, share our lessons learned, and provide recommendations for integrating peers into hospital-based SUD care.

\section{Our Peer Program Description:}

We describe peers included as part of a hospital-based addiction medicine consult team called the Improving Addiction Care Team (IMPACT). IMPACT is comprised of medical providers 
(physicians, a nurse practitioner and physician assistant), social workers, and peer mentors. IMPACT meets patients during the reachable moment of hospitalization, ${ }^{9,12}$ performs an initial assessment; elicits patient-centered goals; may initiate SUD treatment, including pharmacotherapy and behavioral treatment; and provides rapid-access to post-hospital SUD care. ${ }^{13}$

We have implemented IMPACT at Oregon Health \& Science University (OHSU), an urban academic medical center in Portland, Oregon. OHSU is a 556-bed hospital and has 8,070 healthcare employees. Prior to IMPACT, OHSU had no experience including peers in hospital care or, to our knowledge, as part of any clinical team.

Since April 2016, IMPACT has had a total of eight peer mentors. Initially IMPACT included one peer, however IMPACT has since expanded to include three peer positions. Peers' tenure with IMPACT has ranged from one to 26 months. Peers have moved on from IMPACT for a variety of reasons, including promotion to manager at an area nonprofit (1), acceptance to medical school (1), and poor fit/ job performance (3).

The following sections (unique challenges and recommendations) stem from our experience implementing peers as part of IMPACT.

\section{Unique challenges of peer mentors working in hospitals}

Hospital settings present unique challenges, both for peers and for the nurses, physicians, and other hospital staff with whom they work. Potential challenges include the rigid professional hierarchy of hospitals; hospital providers' commonly held negative beliefs towards people with SUD; frequent rotation of providers on and off service; presence of trainees such as students and residents; the fast pace and high demands of hospital care; and high patient acuity, including a focus on end-of-life care or death.

\section{Contrast with traditional hospital roles:}

The peer role is new in hospitals and contrasts starkly with professional roles of physicians, nurses, and social workers. While the hospital hierarchy is based on training, discipline, and specialization, peers' expertise comes from lived experiences. We found that introducing peers raised frequent questions about peers' value as part of the care team, what they do, whether they are paid, and where they belong. Peers may visibly stand out from other hospital staff both in their appearance and their behaviors. A history of SUD may also come with consequences that are stigmatized in healthcare settings, including incarceration, homelessness, mental illness, and other trauma. Peers are open about these experiences, which may cause discomfort or discrimination. The hierarchy may be at times intimidating or infuriating to peers from outside the system.

Professional Boundaries:

Healthcare professionals are taught to maintain clear boundaries and are discouraged from disclosing personal information or developing personal relationships with patients. In contrast, peers disclose their personal histories and prioritize personal connection. For hospital providers, it may be confusing or uncomfortable to see peers acting in the role of a friend. In some 
instances, it may bring up concerns that peers are colluding with patients against providers or the health system.

Goals of hospital care:

There is an inherent tension of hiring peers to achieve SUD and medical treatment outcomes, when peers themselves avoid having an agenda. While hospital providers may engage patients in shared decision-making, providers' goal is to decrease patients' substance use and treat their medical conditions. In contrast, peers' role is not to persuade patients and their relationship with patients is not tied to any specific agenda. Providers may be uncomfortable watching a peer so completely accept patients' choices.

Intensity of hospital environment and patient illness:

Hospital peer mentorship can be an intense role. While caring for sick patients can be distressing for any provider, peers may experience increased stress as this may be their first exposure to acute care, it may be easier to identify with marginalized patients, and because relationships are the foundation for their role. Further, peers are surrounded by conflicts between staff, patients with SUD, family members, and others. The liminal position of peers often thrusts them into the middle of such conflicts. The combination of hospital intensity and all of the above-described challenges can cause stress, burnout, and risk for relapse. ${ }^{10}$

\section{Recommendations}

Given these unique opportunities and challenges, authors outline a series of recommendations for integrating peers into hospital settings. Box 1 includes a summary of suggested guidelines to promote successful integration of peers into hospital care, and can be used as a checklist to guide preparedness and implementation. Authors share details of our experience not to suggest that readers must replicate our approach, but instead as examples that highlight specific challenges and potential solutions. Addressing these kinds of logistics and details are important to the success of peer programs.

Establish a way to finance the peer program, including peer salaries, salary for supervision, and petty cash fund.

Hospital-based peers are paid employees. While in some regions, insurance plans allow for peers to bill for their services, this is not an option in Oregon. This is further complicated in a hospital where there is not typically a mechanism to bill for peer-based services, and where payment is based on a single diagnostic related group (DRG) rate for the entire bundle of hospital care. One approach to funding a peer program that we used for IMPACT was to develop a business case for the full addiction medicine consult service based on length-of-stay and readmission reductions, ${ }^{12}$ and incorporate peer salaries into the full budget analysis.

Authors recommend budgeting for peer salary and for salary to support supervisors' time (approximately 2 hours per week for the first 6 months, then 1 hour/ week). We also recommend having a petty cash fund for peers to purchase small items such as bus tickets or inexpensive items (e.g. journals) that peers can give patients to help them pass down time in the 
hospital. IMPACT labels these costs for "peer engagement" and estimates approximately $\$ 10-20$ per patient.

\section{Clearly define the peer role}

Defining the peer role is particularly important given how different it is from traditional hospital roles. Peers may perform small acts such as bringing a patient something to read or a clean pair of clothes, doing a patients' hair, or accompanying a patient on smoke breaks. Peers often lend a listening ear, which can lessen the isolation that is so often part of peoples' hospital experiences.

Peers can be present during visits with doctors or nurses, or at care conferences, where they can serve to broker communication and culture between patients and providers. Peers often learn new information unknown to the hospital teams. Examples include information about active substance use in the hospital, disclosure about past traumas, knowledge of outstanding warrants, and other deeply personal information (for example, learning that a patient is hospitalized under a false alias).

Peers can also serve a bridging role, escorting patients from the hospital to treatment or arranging in-person meetings to support connection to other community services. This helps provide support at extremely vulnerable times where triggers and relapse risks abound. Peers can bring personal knowledge of community resources when devising care plans. This has included helping patients access housing, community treatment, and navigating the criminal justice system.

Our experience supports that to be effective, peer mentors must be able to work with patients in confidence. Peers are mandated reporters (e.g. in case of child abuse); however, IMPACT peers are not obliged to share private details about patients' substance use or other behaviors. Peers may, however, encourage patients to share private information with providers if they see how it could support their care.

There is practice variation across peer-delivered services as to whether peers take a social history or document in the electronic health record (EHR). In our hospital, peers do not take a formal medical or social history. This is due in part because of barriers within the EHR, which has no mechanism to label notes from nontraditional staff, and in part because IMPACT leadership felt charting responsibilities could make the peer role too clinical and detract from peers' focus on relationship. IMPACT peers do communicate daily (or more) with physician and social work team members who may relay key information in their daily notes. Peers spend only approximately $60 \%$ of their time delivering direct patient service. They spend their remaining time in supervision, participating in meetings with other peers, networking, and participating in hospital team meetings.

\section{Create a home base within the hospital}

Our experience supports the importance of a clinical champion within the hospital to provide peers with a sense of belonging, day-to-day logistical support, and professional support when challenges with patients or staff arise. This champion can help pave the way for peers, being a leading voice for the value of peers, and introducing peers to hospital staff. The champion should have clear channels of communication with the peer supervisor. 
Peers can be embedded as part of an interprofessional addiction medicine consult team such as IMPACT where the physician leader is a champion; however, we believe that peers could function as part of a hospital social work team or primary care team that reaches into the hospital.

Peer participation in daily huddles with other members of the clinical team facilitates regular, close communication and supports collaboration across disciplines. Peer participation in weekly quality improvement meetings elevates the peer voice and the patient voice, and guides fundamental approaches to patient care and systems change. We initially considered having IMPACT peers available during evenings and weekends (when the rest of the team is unavailable); however, we found that peers were more successful if they worked hours when the full team was available in case conflicts arose, and to obtain necessary guidance.

\section{Create a collaborative, structured process for hiring and retaining peers}

Authors recommend engaging hospital leadership early in the process of developing a peer program to foster leadership understanding of the value of peers and get leadership buy-in, which can be helpful in troubleshooting unplanned challenges.

For example, before hiring or introducing peers, the IMPACT physician director and a social work manager convened a meeting with senior leadership from human resources, risk management, and public safety. This meeting described the rationale for peers and allowed all participants to identify potential challenges. The process built a shared commitment, such that when unanticipated problems arose (e.g. our first peer did not pass the OHSU background check), hospital leadership was able to collaborate and problem solve. Authors recommend also including nurse leadership in early meetings.

Given the strong association between SUD and history of incarceration, many peers may have a criminal record. Authors recommend identifying a standard process for peer background checks and suggest hospitals consider honoring an already established standard such as that used by Certified Recovery Mentors (a national certification) or the Department of Human Services/ Child Welfare system.

Authors recommend that hospitals partner with individuals or agencies with experience delivering peer services. Experience and expertise hiring, training, and supervising peers in other physical and behavioral health settings can be highly valuable. While some hospitals may hire peers directly, IMPACT decided to partner with a community peer agency and contract with them to provide peer services.

Authors suggest presenting example challenging case scenarios during the job interview. Such scenarios provide an opportunity to explore applicants' views on medication treatment (e.g. methadone, buprenorphine) and potential candidates comfort navigating complex boundaries. Authors recommend including physicians, social workers, and existing peer mentors (if available) as part of the interview, and explicitly asking about working as part of an interprofessional team. Authors recommend that teams consider offering shadowing opportunities - before extending or accepting a job offer - to allow candidates to see the fast-paced, intense, hospital environment and to allow the hospital team to observe candidates. 
Finally, authors recommend developing processes to identify and support peers who are struggling, and processes to terminate peers who are not a good fit.

\section{Identify peers who are likely to succeed in the role.}

Successful peers have strong communication skills, accountability, the ability adapt to multiple settings (e.g. hospital, community), and flexibility to work with many different people (e.g. peers, physicians, nurses). Peers must be able to tolerate a hospital environment, including working with acutely ill people who may have visible abscesses, IVs, or drains. Peers should be stable in their recovery, typically considered two years of continuous sobriety. Finally, peers with connections to community resources (for example, knowing people who work in community SUD treatment or housing) can bring added value.

In addition to having completed peer certification or equivalent training, authors believe that hospital peers must be open to medication treatment (e.g. buprenorphine, methadone). Within some mutual aid and substance use treatment settings, people can be discouraged from taking medication, perpetuating stigma and harming patients. Authors recommend selecting peers who can embrace harm reduction strategies and who understand that not all people want treatment.

\section{In addition to peer certification, provide initial and ongoing peer training.}

Initial training should include introduction to hospital staff roles, workflows and procedures. Topics might include responsibilities of primary versus consulting teams; roles of intern, resident, and attendings; how to contact a patient's nurse; infection control practices including gloving and gowning; and nuances of hospital care such as the importance of discharging patients early in the day or avoiding extending hospital length of stay unnecessarily. It is important for peers to understand hospital rules for patients, which might include patient expectations for signing on and off the unit, visitor policies, designated smoking areas, and visiting with other patients. Adhering to hospital rules is important to maintaining credibility with hospital staff, and to supporting patients who may struggle with accepting and adhering to such rules. It is also helpful for peers to understand the basics of medical treatment for conditions common in hospitalized adults with SUD. In our experience, some training can happen informally, for example, during daily team huddles. However, planned discussion of difficult scenarios can be helpful (e.g. factors contributing to whether a patient is listed for liver transplant) to guard against an "us" (peers) versus "them" (other healthcare providers) response and allow peers to understand the decisions in the context of healthcare delivery system.

Expectations around confidentiality are important. Peers must protect patients' personal health information (e.g. destroy printed patient lists, utilize secure email) and they are mandated reporters in the case of child abuse. Other information may be more discretionary, but should be discussed up front with peers and the hospital team. For example, patients have shared with peers instances of active use in the hospital, diversion and misuse of medications, and presentation to the hospital under false aliases. Our team elected to give peers the option of keeping this information confidential, with the caveat that peers encourage patients to disclose information when appropriate.

Introduce the peer program to hospital staff. 
In the early stages of a peer program, hospital staff may have frequent questions about peers' role and how peers interact with patients and staff. ${ }^{10}$ IMPACT found newsletters, in-person meetings, and flyers describing the basics of peer role and their scope of work were helpful. Different disciplines preferred different communication strategies (for example, nurses requested posting a flyer in break rooms whereas social workers preferred meeting peers inperson). It was also important to have clear avenues for hospital staff to communicate with IMPACT leadership.

\section{Provide regular, meaningful supervision.}

Authors recommend requiring regular supervision for peers (at least biweekly). If possible, we recommend identifying a peer supervisor who is also in recovery. Supervision should incorporate a space to process emotional responses to work; discuss peers' own recovery; and support to maintain the fidelity of the peer role. It is critical that program leadership understand the importance of supervision and self-care, and that they commit to protecting time needed for this.

\section{Conclusions:}

Peers' lived experience of addiction and its consequences, combined with their distance from medical culture and hierarchy, is at the core of their power - and creates inherent challenges in integrating peers into hospital settings. This guideline outlines practical considerations and potential challenges when integrating peers into hospital-based addictions care, and provides lessons learned from the authors' experience. Hospital providers are generally unfamiliar with peer roles, which contrast starkly with traditional hospital roles. Providers may be concerned that peers are colluding with patients or feel uncomfortable with their non-agenda approach. Peers may feel overwhelmed in a hierarchical and intense hospital environment, and their success in their role depends on individual, team, and organizational factors. By recognizing and addressing potential challenges, we hope to support the rapidly growing interest in integrating peers as part of hospital-based addiction medicine care. 


\section{BOX. Guidelines to Promote Successful Integration of Peers into Hospital Care}

1. Establish a way to finance the peer program, including peer salaries, funding for peer supervision, and a petty cash fund

2. Clearly define the peer role.

3. Create a home base within the hospital setting.

Identify an anchor or clinical champion.

Embed peers within a team. Include peers in team huddles or rounds, and in regular program development and quality improvement meetings.

Provide clear channels of communication between peer supervisor and program leadership.

4. Create a collaborative, structured process for hiring and retaining peers

Engage hospital leadership.

Identify and partner with others experienced working with peers.

Identify standard process for background checks.

Create shadowing opportunities for candidates.

Create processes to identify and support peers who are struggling with the position.

Create processes to terminate peers who are not a good fit.

5. Identify and recruit peers who are likely to succeed in the role.

6. Provide initial and ongoing training to peers, including opportunities to:

Shadow interprofessional hospital staff, including physicians, social workers, and nurses.

Learn occupational health policies and procedures

Learn confidentiality requirements

Learn hospital rules for patients

Learn basics of medical treatment for conditions common in hospitalized patients with SUD

7. Introduce the peer program to hospital staff.

Introduce peers to hospital staff (may include newsletters, in-person meetings, and flyers).

Provide avenues for communication with program leadership.

8. Provide regular, meaningful supervision.

Arrange time for regular supervision (e.g. biweekly). 
Ensure supervision incorporates a space to process emotional responses to work; discuss peers' own recovery; and support to maintain the fidelity of the peer role.

Support opportunities for self-care and communicate its value. 


\section{References}

1. Gagne C, Olivet J, Davis L. Equipping Behavioral Health Systems and Authorities to Promote Peer Specialist/Peer Recovery Coaching Services: Expert Panel Meeting Report. Rockville, MD: Substance Abuse and Mental Health Services Administration, HHS;2012.

2. Jack HE, Oller D, Kelly J, Magidson JF, Wakeman SE. Addressing substance use disorder in primary care: the role, integration, and impact of recovery coaches. Subst Abus. 2017:1-8.

3. Bassuk EL, Hanson J, Greene RN, Richard M, Laudet A. Peer-delivered recovery support services for addictions in the United States: a systematic review. J Subst Abuse Treat. 2016;63:1-9.

4. Reif S, Braude L, Lyman DR, et al. Peer recovery support for individuals with substance use disorders: assessing the evidence. Psychiatr Serv. 2014;65(7):853-861.

5. $\quad$ HB 4143-A, 79th Leg, Regular Sess (Or 2018). Available at: https://olis.leg.state.or.us/liz/2018R1/Downloads/MeasureDocument/HB4 143. Accessed January 23, 2019.

6. Schulman M. Intergrating peers into hospital settings to address substance use: Q\&A with a peer navigator. Center for Health Care Strategies [Webpage]. Available at: https://www.chcs.org/integrating-peers-into-hospital-settingsto-address-substance-use-qa-with-a-peer-navigator/. Accessed January 23, 2019.

7. Chapman S, Blash L, Chan K. The Peer Provider Workforce in Behavioral Health: A Landscape Analysis. San Francisco, CA: UCSF Health Workforce Research Center on Long-Term Care;2015.

8. Substance Abuse and Mental Health Services Administration. Core competencies for peer workers in behavioral health services. 2015. Available at:

https://www.samhsa.gov/sites/default/files/programs campaigns/brss tac s/core-competencies.pdf. Accessed January 22, 2019.

9. Velez CM, Nicolaidis C, Korthuis PT, Englander H. "It's been an experience, a life learning experience": a qualitative study of hospitalized patients with substance use disorders. J Gen Intern Med. 2017;32(3):296-303.

10. Collins D, Alla J, Gregg J, Gullickson J, Nicolaidis C, Englander H. "If it wasn't for him, I wouldn't have talked to them": a qualitative study of peer mentorship for patients with SUD in the hospital setting (in review).

11. Englander H, Collins D, Perry SP, Rabinowitz M, Phoutrides E and Nicolaidis C. "We've learned it's a medical illness, not a moral choice": qualitative study of the effects of a multicomponent addiction intervention on hospital providers' attitudes and experiences. J Hosp Med. 2018;13(11):752-758.

12. Englander $\mathrm{H}$, Weimer $\mathrm{M}$, Solotaroff $\mathrm{R}$, et al. Planning and designing the Improving Addiction Care Team (IMPACT) for hospitalized adults with substance use disorder. J Hosp Med. 2017;12(5):339-342.

13. Englander H, Brandt K, Mahoney S, Brown J, Nydahl A, Weimer M, Gregg J. Tools to support hospital-based addiction care: core components, values, and activities of the Improving Addiction Care Team (IMPACT) J Addict Med. 
